\title{
Correction to: MGUS Predicts Worse Prognosis in Patients with CoronaryArtery Disease
}

\author{
Zhao Xu ${ }^{1} \cdot$ Yifeng Sun ${ }^{1} \cdot$ Tianhong Xu ${ }^{1} \cdot$ Yidan $\mathrm{Shi}^{2} \cdot$ Lifan Liang $^{1} \cdot \mathrm{Peng} \mathrm{Liu}^{1} \cdot \mathrm{Junbo} \mathrm{Ge}^{3}$ \\ Published online: 26 March 2020 \\ (C) Springer Science+Business Media, LLC, part of Springer Nature 2020
}

\section{Correction to: J. of Cardiovasc. Trans. Res. https://doi.org/10.1007/s12265-019-09950-w}

The following are the correction captions for Figures 1-4: Fig. 1 Monoclonal-protein based MGUS screening protocol. MGUS refers to monoclonal gammopathy of undetermined significance; SPEP, serum protein electrophoresis; IFE, immunofixation electrophoresis.

Fig. 2 Kaplan-Meier survival curves for incidence of MACE in CAD patients with and without MGUS.MACE refers to major adverse cardiac events; CAD, coronary artery disease; MGUS, monoclonal gammopathy of undetermined significance
Fig. 3 The nomogram based on the stepwise Cox regression model to predict the outcomes of CAD patients.MGUS refers to monoclonal gammopathy of undetermined significance; eGFR, estimated glomerular filtration rate; MACE, major adverse cardiac events.

Fig. 4 The calibration curve ofMACE-free survival at 1,2 and 3 years for CAD patients. Nomogram-predictedMACE-free probability is plotted on the $\mathrm{x}$-axis; actual MACE-free probability is plotted on the y-axis.

The original article has been corrected.

Publisher's Note Springer Nature remains neutral with regard to jurisdictional claims in published maps and institutional affiliations.
The online version of the original article can be found at https://doi.org/ 10.1007/s12265-019-09950-w

Peng Liu

liu.peng@zs-hospital.sh.cn

1 Department of Hematology, Zhongshan Hospital Fudan University, 180 Fenglin Road, Shanghai 200032, China

2 Department of Statistics and Actuarial Science, University ofWaterloo, Waterloo, Canada

3 Department of Cardiology, Shanghai Institute of Cardiovascular Diseases, Zhongshan Hospital Fudan University, Shanghai, China 